\section{Neutrophil-lymphocyte ratio, pain perception, and disease activity score may serve as important predictive markers for sustained remission in rheumatoid arthritis}

\author{
S. Chandrashekara, A. Rajendran, A. Bai Jaganath, R. Krishnamurthy
ChanRe Rheumatology and Immunology Center, Basaweswaranagar, Bangalore, India \\ S. Chandrashekara, A. Rajendran, A. Bai Jaganath, R. Krishnamurthy
ChanRe Rheumatology and Immunology Center, Basaweswaranagar, Bangalore, India
}

\begin{abstract}
SUMMARY
The implementation of new treatment strategies based on current recommendations has enabled a greater number of patients with rheumatoid arthritis (RA) to achieve remission. However, there are no definite predictors of sustained remission. Moreover, the absence of clear consensus on the time of withdrawal or reduction of treatment further adds to the treatment burden. This pilot study was intended to evaluate the prognostic potential of various RA-related parameters.

All the enrolled subjects $(n=124)$ were clinically evaluated on the basis of various parameters including age, gender, duration of illness before the initiation of disease-modifying anti-rheumatic drugs, tender and swollen joints (28 joints), erythrocyte sedimentation rate (ESR), C-reactive protein (CRP), hemoglobin percentage, lymphocyte count, total white-blood cell counts, and neutrophil to lymphocyte ratio (NLR). Student's t-test and discriminant function analysis were performed. The specificity of all parameters and their best possible cut-off to predict relapse were calculated using the receiver operating characteristic (ROC) analysis.

A significant difference in terms of pain perception, NLR, tendency to have a significant tender joint count and absolute lymphocyte counts was identified between the patients in sustained remission and those in relapse. The ROC analysis indicated that NLR was consistent in predicting remission.

CRP, ESR, and/or disease activity score may not be very effective in differentiating patients with sustainable remission/low disease activity. NLR along with patient's perception of pain may assist in predicting sustained remission.
\end{abstract}

Key words: Neutrophil-lymphocyte ratio; remission; DAS; VAS; CRP; ESR; rheumatoid arthritis; disease-modifying antirheumatic drugs; DMARDs.

Reumatismo, 2015; 67 (3): 109-115

\section{INTRODUCTION}

$\mathrm{R}$ heumatoid arthritis (RA) is a chronic inflammatory disorder marked by variable cycles of remissions and relapses. In recent years, the focus on the outcome of RA has shifted significantly from symptomatic relief and reduction of erosion/ deformities to the importance of minimum disease activity and remission. This change was dictated by the evidence that the level of disease activity and the duration of remission influence the subsequent progression of the radiographic damage in RA (1). Hence, the current recommendations have incorporated the concept of treat-to-target approach to achieve low disease activity (LDA)/remission (2). However, there is no consensus in the recommendations about the time of withdrawal or reduction of disease-modifying anti-rheumatic drugs (DMARDs). The current treatment strategies, including early treatment with DMARDs, use of biologicals and newer DMARDs, and disease activity score (DAS)-driven treat-to-target protocols of RA increased the number of patients achieving remission (3). Various studies indicated that a certain proportion of patients may reach remission regardless of the drugs administered to obtain disease control (4). Several studies are currently ex-
Corresponding author:

S. Chandrashekara,

ChanRe Rheumatology and Immunology Center, 149, 15th main, NHCL water tank Road, 4th Block, 3rd stage, Basaweswaranagar, Bangalore, India

E-mail: chandrashekara_s@yahoo.com 
ploring whether, after achieving remission, it is necessary to continue with an intense regimen either with expensive biologics or DMARD combinations. Flare was reported in approximately $26-40 \%$ of patients depending on the number of follow-up years, the type of drugs used to induce remission, and the minimum DMARD dosage used for maintenance. These findings underscore the need for markers to predict the sustainability of remission in RA patients.

The available predictors of remissions can be categorized as modifiable and nonmodifiable. The genetic make-up, patient age, and, to some extent, the delay in the initiation of the appropriate treatment are considered as non-modifiable factors. The delay in seeking medical intervention in a large cohort is modifiable through education, but it is not modifiable at the individual level once the patient sought medical attention. The modifiable factors include treatment regimen, targetable disease activity parameters, and lymphocyte count. Moreover, an effective DMARD treatment contributes to a reduction in inflammation, which is also reflected in the neutrophil to lymphocyte (NLR) ratio. DMARDs can be modified to achieve targetable outcome variables like DAS score and lymphocyte count. The changes in lymphocyte count and the ratio depend on disease activity and the influence of DMARDs on lymphocytes $(5,6)$.

Based on this assumption, we hypothesized that the identification of a specific treatment target for disease monitoring in the routine practice to predict sustained remission may serve as an important marker for discontinuing DMARDs. Normalization of inflammatory markers may function as an effective predictor of sustained remission, since DAS-C-reactive protein (CRP), joint count, and CRP may attain a flooring effect with a low disease activity (7). Our preliminary study suggested a weaker relationship with clinical parameters than with laboratory parameters in the presence of low disease activity (8). After attaining clinical remission, markers indicating the harmonization of the immune response and sensitive parameters of inflammation can aid in determining the persistence of remission. NLR has emerged as one of the highly sensitive measures of inflammation in the field of oncology, cardiology, diabetes, and infectious diseases (9-12). It also proved to be a useful indicator in certain inflammatory diseases like ankylosing spondylitis, active familial Mediterranean fever, and Henoch-Schönlein purpura (13-15). Neutrophils mirroring innate immunity and lymphocytes reflecting adaptive immunity should serve as a suitable marker for RA remission. In this study, we used DAS 28 (3) CRP, since we found a significant inconsistency in the visual analogue scale (VAS) reported by our patients. Evidence in the literature suggest that DAS 28 (3) CRP is a good measure to estimate remission (16). The present prospective observational study analyzed the probable predictors of sustained LDA/remission within the measurable parameters of disease activity, including NLR.

\section{PATIENTS AND METHODS}

The patients who attended our out-patient facility between March and June 2013 and fulfilled the American College of Rheumatology/European League Against Rheumatism (ACR/EULAR) 2010 classification criteria for RA were included in the study. Among these patients, those who fulfilled the remission criteria with a DAS 28 (3) CRP score below 2.6 were recruited for this study. They were evaluated at the $3^{\text {rd }}$ and $6^{\text {th }}$ month during the follow-up period within 2 weeks before and after the expected date. The following demographic and clinical characteristics were assessed at the time of recruitment: age, gender, duration of illness before the initiation of DMARD, tender (TJC) and swollen (SJC) joints (28 joints), erythrocyte sedimentation rate (ESR), CRP, hemoglobin percentage $(\mathrm{Hb} \%)$, lymphocyte count, total whiteblood cell (WBC) counts, and NLR. The exclusion criteria considered were: noncompliance or no willingness to participate in the study, inability to comprehend the analogue scale and other tools, presence of severely deformed arthritis belonging 
Table I - Clinical and demographic characteristics of the study subjects.

\begin{tabular}{|l|c|c|c|}
\hline \multirow{2}{*}{ Demographic characteristics } & \multicolumn{2}{|c|}{ Classification based on DAS-CRP score } \\
\cline { 2 - 4 } & $\begin{array}{c}\text { In remission } \\
\text { ( } \mathbf{n}=\mathbf{6 5})\end{array}$ & $\begin{array}{c}\text { In relapse } \\
(\mathbf{n}=\mathbf{2 3})\end{array}$ & P value \\
\cline { 2 - 4 } & Mean \pm SD & Mean \pm SD & P(T<=t) two-tail \\
\hline Age (in years) & $48.5 \pm 10.8$ & $51.2 \pm 11.4$ & 0.32 \\
\hline Gender (M/F) (chi-square test) & $9 / 56$ & $3 / 20$ & 0.92 \\
\hline Duration of illness (months) & $52.8 \pm 43.6$ & $57.2 \pm 43.9$ & 0.68 \\
\hline VAS (in mm) & $9.7 \pm 11.1$ & $16.9 \pm 9.6$ & $0.006^{*}$ \\
\hline SJC & $0.03 \pm 0.17$ & $0.08 \pm 0.28$ & 0.27 \\
\hline TJC & $0.47 \pm 1.16$ & $0.95 \pm 1.10$ & $0.08^{\circ}$ \\
\hline Baseline CRP (mg/L) & $2.86 \pm 2.3$ & $3.43 \pm 2.16$ & 0.29 \\
\hline Baseline ESR (mm/h) & $24.8 \pm 14.6$ & $29.0 \pm 19.1$ & 0.27 \\
\hline Neutrophil $(\%)$ & $60.5 \pm 8.09$ & $63.1 \pm 8.57$ & 0.20 \\
\hline Lymphocyte (\%) & $28.5 \pm 7.12$ & $25.4 \pm 7.28$ & $0.07^{\circ}$ \\
\hline NLR & $2.32 \pm 0.87$ & $2.87 \pm 1.53$ & $0.04^{*}$ \\
\hline Total lymphocyte count (cells/cu mL) & $6795.6 \pm 1873.8$ & $6476.9 \pm 1579.9$ & 0.46 \\
\hline Absolute lymphocyte count (cells/cu mm) & $1922.0 \pm 644.0$ & $1646.2 \pm 650.7$ & $0.08^{\dagger}$ \\
\hline VHB (mg/dL) & $12.3 \pm 1.51$ & $12.1 \pm 1.40$ & 0.54 \\
\hline DAS-CRP & $1.69 \pm 0.45$ & $1.76 \pm 0.37$ & 0.52 \\
\hline DAS-ESR & $2.59 \pm 063$ & $2.72 \pm 0.64$ & 0.41 \\
\hline
\end{tabular}

DAS, disease activity score; CRP, C-reactive protein; SD, standard deviation; VAS, visual analogue scale; SJC, swollen joint count; TJC, tender joint count; NLR, neutrophil to lymphocyte ratio; VHB, xxxxx; ESR, erythrocyte sedimentation rate. *Significant; ${ }^{\circ}$ Borderline significant.

to stage 4 Steinbrocker functional classification, and additional co-morbidities i.e. stroke, depression, fibromyalgia and overlapping connective tissue diseases, concomitant use of oral steroids and use of parenteral steroids within a month from the time of screening. Patients who required a change in the DMARD treatment due to adverse events were also excluded. The recruited patients underwent a routine assessment by a single physician. The joint evaluation was done by an independent joint assessor (IJA). Pain perception by VAS (100 mm scale), ESR (Westergren), and CRP was also determined. IJA also assisted in VAS evaluation. The study was reviewed and approved by the local Institutional ethical committee.

\section{Statistical analysis}

Student's $t$-test was performed using Microsoft Excel to compare the differences between the continuous variables, while the chi-square test was used for categorical variables. The remission was scored as 0 and the relapse as 1 . A discriminant function analysis was carried out for all parameters using online VassarStats software. The receiver operating characteristic (ROC) analysis was performed using MedCalc to identify the specificity of all parameters and their best possible cut-off to predict relapse.

\section{RESULTS}

Out of the 135 patients screened, 124 qualified for the study. Ten patients received parenteral or oral steroids and a screen failure was reported in 1 patient. Out of 124 patients, 110 were on oral methotrexate $15-25 \mathrm{mg} /$ week, 38 on leflunomide, and 120 on hydroxychloroquine. All participants were on combination treatments and 34 were taking the three-drug combination. None had received biologicals. The 
data of the 88 patients who completed the 2 follow-up visits were considered for the analysis. Their demographic details are reported in Table I. Out of 124 patients, 6 were excluded, because the DMARD dosage had to be changed due to elevated liver enzymes and 30 patients missed the first follow-up visit after 3 months. Eight out of 30 patients who attended the second follow-up after 6 months attained disease remission. However, they were not considered for the study, since their first followup status was unclear.

A significant difference was identified between the patients in sustained remission and in relapse with regard to pain perception, NLR, tendency to have a significant TJC, and percentage/absolute lymphocyte counts. The discriminant function analysis indicated that VAS, NLR, absolute lymphocyte count, and TJC significantly influenced the patient classification. The

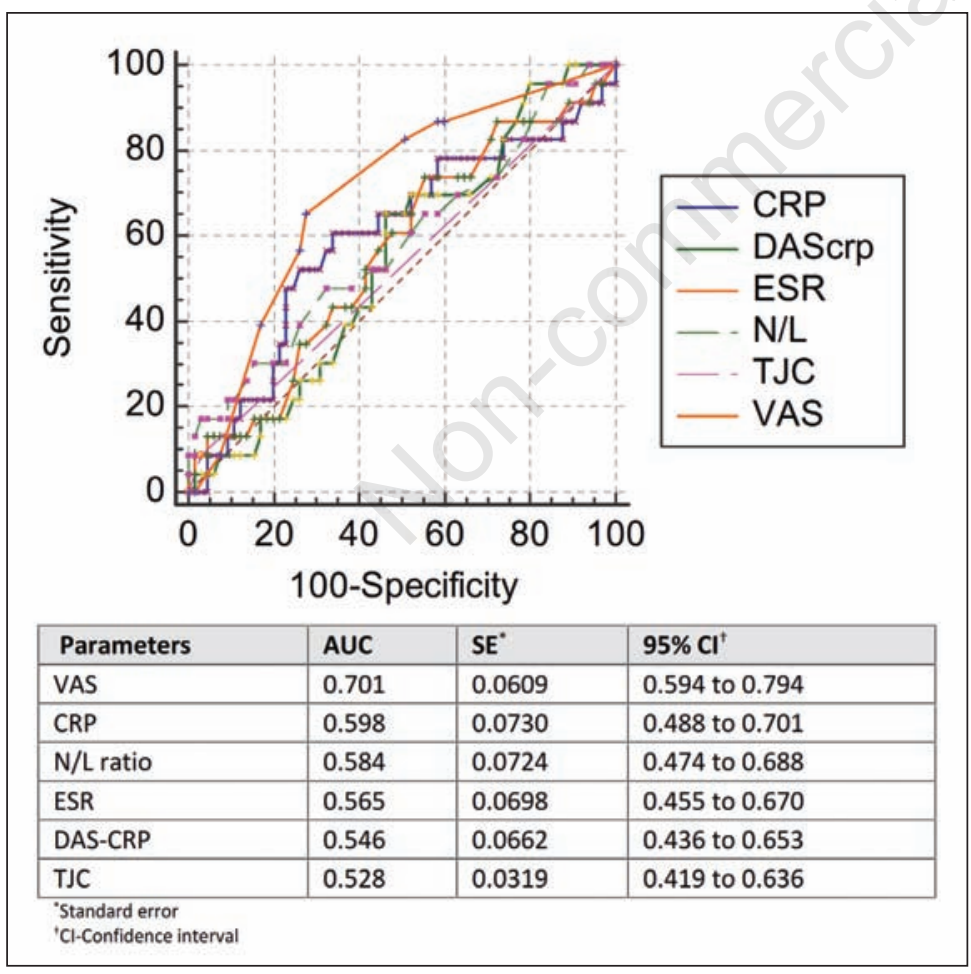

Figure 1 - Receiver operating characteristic curve of the six significant parameters. CRP, C-reactive protein; DAScrp, disease activity score-CRP; ESR, erythrocyte sedimentation rate; N/L, neutrophil to lymphocyte; TJC, tender joint count; VAS, visual analogue scale.
TJC, NLR, and VAS positively predicted relapse, while the lymphocyte count predicted it negatively (in remission). There was no significant influence of duration of disease, treatment, and age in predicting the outcome. The ROC analysis (Figure 1) showed that these parameters are not very strong independent predictors, since their areas under the curve (AUC) were only slightly greater than 0.5 . However, CRP was found to be slightly better than NLR. The best cut-off values with a positive predictive value greater than 1.5 are indicated in Table II.

Considering the ROC analysis of NLR, DAS-CRP, and CRP, NLR was found to be consistent in predicting remission. The AUC was greater for VAS, followed by CRP and NLR. However, DAS-CRP and CRP had no discriminating potential, and the positive predictive value was better for VAS followed by CRP (Table III).

\section{DISCUSSION}

The present study suggests that NLR, CRP, and DAS-CRP are the most effective predictors of relapse, in addition to the presence of tender joint and pain perception by the patient. The association of TJC and pain confirms the previous observations that the presence of signs of measurable inflammation is one of the important predictors of relapse. The signs of residual inflammation of RA either indicated by Power Doppler ultrasound or by the examination of TJC and VAS are negative predictors of sustained remission (17). The discriminant function analysis as well as the difference between the remission group and the sustained remission group did not indicate the discriminating potential of SJC, DAS score, and CRP. Since SJC, CRP, and DASCRP are interdependent and correlated with each other, DAS-CRP can be considered as a marker representing both TJC and SJC. Moreover, TJC and SJC are included in the composite DAS score and have no discriminatory strength in the state of remission, as they are affected by a flooring effect (7). However, the ROC analysis demonstrated that the patients in remission/relapse can 
Table II - The best cut-off value of four selected parameters for predicting relapse.

\begin{tabular}{|l|l|l|l|l|l|l|l|l|l|}
\hline Parameters & Criterion & Sensitivity & $95 \% \mathrm{Cl}$ & Specificity & $\mathbf{9 5 \% ~ C l}$ & $\begin{array}{l}\text { +ve } \\
\text { Likelihood } \\
\text { ratio }\end{array}$ & $\mathbf{9 5 \% ~ C l}$ & $\begin{array}{l}\text {-ve } \\
\text { Likelihood } \\
\text { ratio }\end{array}$ & 95\% Cl \\
\hline DAS-CRP & $>1.67$ & 60.87 & $38.5-80.3$ & 53.85 & $41.0-66.3$ & 1.32 & $0.9-2.0$ & 0.73 & $0.4-1.3$ \\
\hline CRP & $>2.9$ & 60.87 & $38.5-80.3$ & 63.08 & $50.2-74.7$ & 1.65 & $1.0-2.6$ & 0.62 & $0.4-1.1$ \\
\hline VAS & $>10$ & 65.22 & $42.7-83.6$ & 72.31 & $59.8-82.7$ & 2.36 & $1.4-3.9$ & 0.48 & $0.3-0.9$ \\
\hline NLR & $>2.9$ & 30.43 & $13.2-52.9$ & 80.00 & $68.2-88.9$ & 1.52 & $0.7-3.3$ & 0.87 & $0.6-1.2$ \\
\hline
\end{tabular}

$\mathrm{Cl}$, confidence interval; DAS, disease activity score; CRP, C-reactive protein; VAS, visual analogue scale; NLR, neutrophil to lymphocyte ratio.

be better classified by CRP than by DAS score. This is consistent in particular when CRP values are around $0.3 \mathrm{mg} / \mathrm{dL}$. However, the likelihood ratio is very inconsistent for both higher and lower values of CRP. The ESR did not show much discriminatory predictability as shown in our previous observation that CRP overlaps and correlates with clinical disease activity better than ESR (8). Patient's perception of pain and TJC demonstrated a good discriminating strength, but TJC may not be a useful measure, as it may remain zero after remis- sion. The study results also demonstrated the usefulness of the VAS pain scale, which is a subjective assessment and one of the reliable measures of disease activity in differentiating the groups (18). Although the measure showed consistency in discriminating the status of inflammation even at the lowest level, its reliability in the presence of fibromyalgia is a concern, as the use of analgesics/antipsychotics may alter pain perception.

NLR, which demonstrated significant differences between the groups, had a better

Table III - Standardized canonical discriminant function coefficients of all the variables evaluated.

\begin{tabular}{|l|c|}
\hline Parameters evaluated & $\begin{array}{c}\text { Function } \\
\text { 1 = relapse }\end{array}$ \\
\hline Age & 0.082 \\
\hline Male & 0.070 \\
\hline Duration of illness (months) & 0.164 \\
\hline VAS & 0.757 \\
\hline SJC & 0.193 \\
\hline TJC & 0.332 \\
\hline CRP & 0.381 \\
\hline ESR & -0.162 \\
\hline Neutrophil & -0.367 \\
\hline Lymphocytes & -0.818 \\
\hline NLR & 0.701 \\
\hline Total WBC count & -0.872 \\
\hline Absolute count of lymphocyte & 0.842 \\
\hline Hb\% at remission & -0.467 \\
\hline DAS 28-(3)CRP at baseline & -0.055 \\
\hline DAS 28-(3)ESR at baseline & -0.303 \\
\hline
\end{tabular}

VAS, visual analogue scale; SJC, swollen joint count; TJC, tender joint count; CRP, C-reactive protein; ESR, erythrocyte sedimentation rate; NLR, neutrophil to lymphocyte ratio; WBC, white-blood cell; $\mathrm{Hb}$, hemoglobin; DAS, disease activity score. 
discriminatory power compared to other markers of inflammation. The ROC analysis indicated a consistently high sensitivity and specificity. The best cut-off of NLR at which a good specificity was achievable in predicting relapse was 2.9 .

This is higher than the normal cut-off value of 2. Evidence in the literature supports the use of elevated NLR as a prognostic indicator in different clinical scenarios of chronic inflammation $(19,20)$. Some studies suggest that it may indeed reflect disease activity in RA and other diseases. Studies of predictors of flare or failed sustained remission reported that the signs of inflammation like persisting ultrasound signs of synovitis, such as power Doppler signals of vascularity, and absence of deep remission indicate an early relapse $(17,21)$. Our study confirms that the signs or laboratory parameters of inflammation are significant in predicting relapse.

However, in order to decide whether to discontinue treatment, measures need to be more specific than sensitive. In this respect, NLR seems to be a good single predictor of sustained remission. However, more longterm follow-up studies are required to evaluate the usefulness of these markers and the possible complications of DMARDs withdrawal.

This study was prospective, but not adequately powered due to a $20 \%$ dropout rate. Since there was no ethical committee approval for the subsequent step of DMARD withdrawal, we could not investigate whether the predictors evaluated indicate a sustainable remission even after drug discontinuation. The committee suggested we undertake such studies after proving the predictability of sustained remission. The study was carried out only for a period of 6 months, therefore our results cannot be generalized for long-term remission. Additional long-term studies characterizing NLR, CRP, and other disease parameters are required to establish the usefulness of these measures in predicting long-term remission. If confirmed, NLR may serve as a useful and cost-effective measure in predicting remission, in addition to other routine parameters.
The ROC analysis of the present study suggests that DAS-CRP score below 1.6 has a greater predictive value for sustained remission and supports the concept of targeting deep remission.

\section{CONCLUSIONS}

CRP, DAS-CRP, NLR, and patient's perception of pain may serve as effective measures to predict sustained remission and low disease activity. Further research should focus on determining the optimal target values of these parameters to identify patients achieving sustained remission.

Funding: the research work is funded by ChanRe Rheumatology and Immunology Center, Bangalore, India, where the authors work.

Acknowledgments: the authors are grateful to Research Assist (http://www.research-assist.com) for assisting in editing and other secretarial support.

Disclosure: the authors report no conflicts of interest in this work

\section{REFERENCES}

1. Aletaha D, Funovits J, Breedveld FC, Sharp J, Segurado O, Smolen JS. Rheumatoid arthritis joint progression in sustained remission is determined by disease activity levels preceding the period of radiographic assessment. Arthritis Rheum. 2009; 60: 1242-9.

2. Gaujoux-Viala C, Gossec L, Cantagrel A, Dougados M, Fautrel B, Mariette X, et al. Recommendations of the French Society for Rheumatology for managing rheumatoid arthritis. Joint Bone Spine. 2014; 81: 287-97.

3. Barnabe C, Homik J, Barr SG, Martin L, Maksymowych WP. The effect of different remission definitions on identification of predictors of both point and sustained remission in rheumatoid arthritis treated with anti-TNF therapy. J Rheumatol. 2014; 41: 1607-13.

4. van der Woude D, Visser K, Klarenbeek NB, Ronday HK, Peeters AJ, Kerstens PJ, et al. Sustained drug-free remission in rheumatoid arthritis after DAS-driven or non-DAS-driven therapy: a comparison of two cohort studies. Rheumatology (Oxford). 2012; 51: 1120-8.

5. Mercan R, Bitik B, Tufan A, Bozbulut UB, Atas N, Ozturk MA, et al. The association 
between neutrophil/lymphocyte ratio and disease activity in rheumatoid arthritis and ankylosing spondylitis. J Clin Lab Anal. 2015 [Epub ahead of print].

6. Chandrashekara S. Pharmacokinetic consideration of synthetic DMARDs in rheumatoid arthritis. Expert Opin Drug Metab Toxicol. 2013; 9: 969-81.

7. Chandrashekara S, Sachin S. Measures in rheumatoid arthritis: are we measuring too many parameters. Int J Rheum Dis. 2012; 15 : 239-48.

8. Chandrashekara S, Renuka P, Suresh KP. ESR or CRP, which inflammatory measure can accurately replace clinical measures in rheumatoid arthritis? Indian J Rheumatol. 2012; 7: 69-73.

9. Dirican A, Kucukzeybek BB, Alacacioglu A, Kucukzeybek Y, Erten C, Varol U, et al. Do the derived neutrophil to lymphocyte ratio and the neutrophil to lymphocyte ratio predict prognosis in breast cancer? Int J Clin Oncol. 2015; 20: 70-81.

10. Chen J, Chen MH, Li S, Guo YL, Zhu CG, $\mathrm{Xu} \mathrm{RX}$, et al. Usefulness of the neutrophilto-lymphocyte ratio in predicting the severity of coronary artery disease: a Gensini score assessment. J Atheroscler Thromb. 2014; 21: 1271-82.

11. Afsar B. The relationship between neutrophil lymphocyte ratio with urinary protein and albumin excretion in newly diagnosed patients with type 2 diabetes. Am J Med Sci. 2014; 347: 217-20.

12. Bekdas M, Goksugur SB, Sarac EG, Erkocoglu M, Demircioglu F. Neutrophil/lymphocyte and C-reactive protein/mean platelet volume ratios in differentiating between viral and bacterial pneumonias and diagnosing early complications in children. Saudi Med J. 2014; 35: 442-7.

13. Gökmen F, Akbal A, Resorlu H, Gökmen E, Güven M, Aras AB, et al. Neutrophil-lympho- cyte ratio connected to treatment options and inflammation markers of ankylosing spondylitis. J Clin Lab Anal. 2015; 29: 294-8.

14. Celikbilek M, Dogan S, Akyol L, Borekci E, Zararsiz G, Kozan M, et al. Neutrophil-lymphocyte ratio in patients with familial Mediterranean fever. J Clin Lab Anal. 2015; 29: 803.

15. Makay B, Gücenmez ÖA, Duman M, Ünsal E. The relationship of neutrophil-to-lymphocyte ratio with gastrointestinal bleeding in HenochSchonlein purpura. Rheumatol Int. 2014; 34 : 1323-7.

16. Balsa A, Carmona L, González-Alvaro I, Belmonte MA, Tena X, Sanmartí R, et al. Value of Disease Activity Score 28 (DAS28) and DAS28-3 compared to American College of Rheumatology-defined remission in rheumatoid arthritis. J Rheumatol. 2004; 31: 40-6.

17. Saleem B, Brown AK, Quinn M, Karim Z, Hensor EM, Conaghan P, et al. Can flare be predicted in DMARD treated RA patients in remission, and is it important? A cohort study. Ann Rheum Dis. 2012; 71: 1316-21.

18. Chandrashekara S, Syed M, Swapna R. Is three selected parameters adequate to monitor rheumatoid arthritis? Clin Rheumatol. 2007; 26: 911-4.

19. Ozler GS, Gunak G. Neutrophil-lymphocyte ratio: a new predictive and prognostic factor in patients with Bell palsy. J Craniofacial Surg 2014; 25: 944-5.

20. Makay B, Gucenmez OA, Duman M, Ünsal E. The relationship of neutrophil-to-lymphocyte ratio with gastrointestinal bleeding in Henoch-Schonlein purpura. Rheum Int. 2014; 34: 1323-7.

21. Tanaka Y, Hirata S, Kubo S, Fukuyo S, Hanami K, Sawamukai N, et al. Discontinuation of adalimumab after achieving remission in patients with established rheumatoid arthritis: 1-year outcome of the HONOR study. Ann Rheum Dis. 2015; 74: 389-95. 